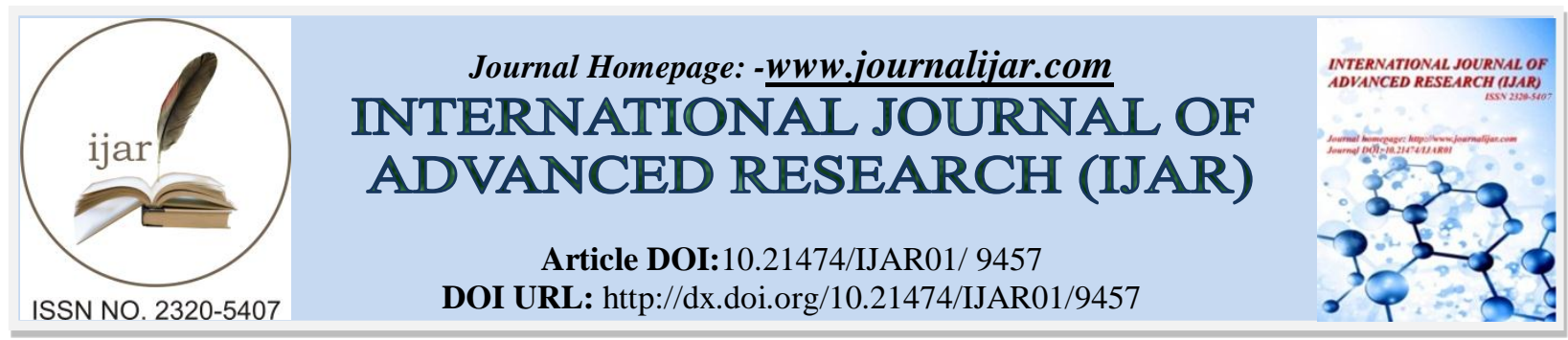

RESEARCH ARTICLE

\title{
COMPARISON OF PREGNANCY RATE AMONG DIFFERENT IVF/ICSI PROTOCOLS IN WOMEN WITH PCOS.
}

Nada Flayyih Hasan AL-Aboudy ${ }^{1}$ and Mohammed Sattar Maroof ${ }^{2}$ and Eqbal Loaibi ${ }^{1}$.

1. Kamal AL-Samarai Hospital, center of fertility and IVF, Baghdad /Iraq.

2. Medical City Center, Baghdad /Iraq.

\section{Manuscript Info \\ Manuscript History \\ Received: 26 May 2019 \\ Final Accepted: 28 June 2019 \\ Published: July 2019}

Key words:-

PCOS, IVF protocols.

\begin{abstract}
Objective: To assess the pregnancy rate after using of different IVF/ICSI protocols in patients with PCOS.

Study design: A total of 55 infertile women with Polycystic ovary syndrome undergoing controlled ovarian hyperstimulation for intracytoplasmic sperm injection cycle were prospectively recruited for this study in High Institute of Infertility Diagnosis and Assisted Reproductive Technology / AL-Nahrian University and Kamal ALSamarai Hospital, center of fertility and IVF (Baghdad/Iraq) during the period from December 2015 to the end of April 2017.The fifty five PCOS women were divided into three groups: Twenty five (25) infertile women have had PCOS and young age undergone long Agonist protocol for ICSI cycle, Fifteen (15) infertile women have had PCOS who young age and have risk of OHSS (AMH $>6 \mathrm{ng} / \mathrm{ml}$ or had previous history of hyper-response to ovarian stimulation protocol) undergone Antagonist protocol for ICSI and Fifteen (15) infertile women have had PCOS and age between 35-40 years old undergone short Agonist protocol for ICSI. In all patients B-HCG measure at day 14 after embryo transfer.

Results: pregnancy rate was significantly higher in the PCOS patients underwent short agonist protocol (40\%) compared to the PCOS patients underwent Antagonist protocol (20\%) and to PCOS patients underwent long agonist protocol $(16 \%)(P=0.0028)$. In this study, the percentage of pregnancy in PCOS group was $(23.6 \%)$.

Conclusion: It was concluded from the present study that the percentage of pregnancy was higher in polycystic ovary syndrome women more than 35 years old underwent short agonist protocol than in other ICSI/IVF protocols.
\end{abstract}

Copy Right, IJAR, 2019,. All rights reserved.

\section{Introduction:-}

Polycystic ovary syndrome (PCOS) is one of the most common endocrinopathies and metabolic abnormalities, affect 10-15\% of women of reproductive age was initially described by Stein and Leventhal in 1935, characterized by ovulatory dysfunction, biochemical and/or clinical hyperandrogenism including hirsutism, alopecia, obesity and polycystic ovarian morphology including the presence of hyper vascularized androgen secreting stroma and enlarged 
ovaries with multiple small follicles $2-8 \mathrm{~mm}$ in diameter ${ }^{(1)}$. It may have complex effects on the ovulation, oocyte quality and the endometrium contributing to a lower fertility potential ${ }^{(2)}$. Different ovarian stimulation protocols have been used to optimize the results of IVF treatments ${ }^{(3)}$ like Gonadotropin releasing hormone agonists (GnRHa) protocols and Antagonist protocol. In the long protocols (with GnRHa started either in the midluteal phase or in the early follicular phase) gonadotropin administration is delayed until pituitary desensitization has been achieved, usually 2-3 weeks. Long protocol of GnRH agonist was better than other protocols because long protocol produce a cohort of follicle that grow simultaneously and this allows retrieval of more oocytes, production of more embryos with ultimate better pregnancy rate ${ }^{(4)}$.

Short agonist protocol is the treatment of choice for patients with diminished ovarian reserve (poor responders) to avoid excessive pituitary suppression. In those patients, GnRH-a may be administered on day 2 or 3 of menstruation for pituitary down regulation. Duration of administration continue for 8-12 days till to ovulation induction that done with $\mathrm{HCG}^{(5)}$.

In the early 2000s, the introduction of GnRH antagonists in IVF was accomplished by five large randomized controlled trials (RCTs) which compared GnRH antagonists with the long GnRH agonist protocol. The metaanalysis of these trials showed that a lower requirement for gonadotrophins, a reduced length of treatment and a dramatic reduction in the duration of analogue treatment was present in the GnRH antagonist group. However, a significantly lower (5\%) clinical pregnancy rate compared with the agonist group has resulted in a lower acceptance of GnRH antagonists in ovarian stimulation and has stimulated research for optimizing the existing GnRH antagonist protocols for IVF. Moreover, a tendency for a lower occurrence of ovarian hyperstimulation syndrome (OHSS) in the antagonist group has suggested that this form of treatment is worth evaluating in patients at high risk for OHSS, such as PCOS ${ }^{(6)}$.

\section{Subjects, Materials and Methods:-}

A prospective study conducted in High Institute of Infertility Diagnosis and Assisted Reproductive Technology / AL-Nahrian University and Kamal AL-Samarai Hospital, center of fertility and IVF (Baghdad/Iraq) during the period from December 2015 to the end of April 2017.Ninety infertile couples (PCOS and controlled) have been enrolled in this study and enter their ICSI cycle. Informed consent of all patients was taken before inclusion in the study. All infertile couples were subjected to a full history taking, complete general and gynecological examination and full infertility investigations including: husband's seminal fluid analysis, hormonal assay, trans-vaginal ultrasound and hystrosalpingography for evaluation of uterine cavity and tubal patency and/or laproscopy for evaluation of tubal patency and exclusion of pelvic pathology. Fifty five women (55) with PCOS were included selected from those who undergoing $\mathrm{COH}$ for ICSI cycle. These (55) women were divided into three groups: I. Twenty five (25) infertile women have had PCOS and young age undergone long Agonist protocol for ICSI cycle. II. Fifteen (15) infertile women have had PCOS who young age and have risk of OHSS (AMH > 6ng/ml or had previous history of hyper-response to ovarian stimulation protocol) undergone Antagonist protocol for ICSI. III. Fifteen (15) infertile women have had PCOS and age between 35-40 years old undergone short Agonist protocol for ICSI. The diagnosis of PCOS depend on fulfilling at least two of three criteria based on the Rotterdam ESHRE/ASRMS sponsored PCOS consensus workshop group ${ }^{(7) . ~ R o t t e r d a m ' s ~ c r i t e r i a ~ o f ~ P C O S ~ w h i c h ~ w a s ~ b a s e d ~}$ on: Typical picture of polycystic ovaries on ultrasonography (ten or more follicles in each ovary, each follicle measuring 2-9 $\mathrm{mm}$ in diameter). 2- Anovulation or Oligo-ovulation. 3- Hyperandrogenism; clinical or biochemical. Clinically hyperandrogenism manifested as a hirsutism, acne and biochemically as elevated serum testosterone level.

First group of PCOS patients(25) were enrolled in long protocol type of IVF/ICSI cycle, an ultrasound examination was performed in order to exclude those women with ovarian cyst and assess the endometrial thickness and started on day 21 of the previous menstrual cycle (mid-luteal) with a daily administration of subcutaneous injection of GnRH-a, triptorelin (Decapeptyl®; $0.1 \mathrm{mg}$ Ferring Co, Kiel, Germany) ${ }^{\circledR}$ for pituitary down-regulation and desensitization and continue till the day of HCG administration. When pituitary down-regulation was achieved (menstruation occur, E2 level reaching $<50 \mathrm{pg} / \mathrm{ml}$ and endometrial thickness was $\leq 2-3 \mathrm{~mm}$ on ultrasound examination ${ }^{(8)}$ ovarain stimulation started with recombinant human follicle stimulating hormone (rhFSH) (Gonal F, Merck ® 75 IU of FSH activity per ampoules) by daily subcutaneous injection in a dose of $150 \mathrm{IU}$. The follicle growth and the doses of Gonal-F® were monitored by trans-vaginal ultrasound (cycle day 5 and subsequent scan were done every 2-3 days as required) and by serum E2 level (day 6-8 of (Gonal-F®) injection and till the day of HCG administration) ${ }^{(9)}$. When either two or three lead follicles have reached $17-18 \mathrm{~mm}$ ovulation induction was 
induced by the administration of recombinant HCG (rhCG 6500 IU, Ovitrelle ${ }^{\circledR}$; Merck, Italy) subcutaneously ${ }^{(10)}$. Second group (15) infertile couples in whom PCOS women were undergoing antagonist protocol, started daily administration of 150 IU of (Gonal-F®) injection subcutaneously from day two of menstrual cycle. GnRH antagonist (Cetrorelix) is usually given in a dose of $0.25 \mathrm{mg}$ daily when the leading follicle reaches a certain size by ultrasound monitoring $(12-14 \mathrm{~mm})$. The antagonist is continued together with the (Gonal-F®) stimulation until an adequate response is obtained and HCG injection requird for ovulation induction ${ }^{(11) .}$ Third group (15) infertile couples in whom PCOS women were undergoing short agonist protocol, which started on day two of menstrual cycle with a daily administration of subcutaneous injection of GnRH-a, triptorelin (Decapeptyl®; $0.1 \mathrm{mg}$ Ferring Co, Kiel, Germany) ${ }^{\circledR}$.ovarain stimulation started on day three of menstrual cycle with daily administration of $\geq$ $225 \mathrm{IU}$ of (Gonal-F®) injection subcutaneously depending on the women's age and previous response of ovulation induction.. Decapeptyl ${ }^{\circledR}$ is continued together with the (Gonal-F®) stimulation until an adequate response is obtained and HCG injection requird for ovulation induction ${ }^{(12)}$. The oocytes were retrieved by transvaginal ultrasound-guided follicle aspiration 34-36 h after HCG administration. Sperm preparation and IVF/ICSI were performed and embryo transfers were carried out 2- 3 days after oocyte retrieval. Luteal phase support was done by giving $400 \mathrm{mg} / \mathrm{bid}$ of vaginal progesterone (Cyclogest ${ }^{\circledR}$, Actavis). Serum $\beta \mathrm{hCG}$ levels were tested two weeks after embryo transfer.

\section{Statistical analysis:}

The Statistical Analysis System- SAS (2012), version 9 was used to evaluate effect of different factors in study parameters. Numeric variables were expressed as mean \pm standard error $(\mathrm{SE})$, while nominal variables were expressed as number and percentage. Chi-square test was used to significant comparison between percentage and least significant difference -LSD test (ANOVA) was used to significant comparison between means. Pearson's correlation coefficient was used to evaluate correlation between numeric variables ${ }^{(13)}$. The differences between values were considered statistically significant at the level of $(\mathrm{p}<0.05)$ and highly significant at the level of $(\mathrm{p}<0.01)$.

\section{Results:-}

Distribution of Study According to Age /per Group of PCOS

In present study only one woman $(4.00 \%)$ from PCOS patients with long protocol and no patient $(0 \%)$ with antagonist or short protocol below age of twenty. The statistical analysis showed no significant $(\mathrm{P}<0.05)$. While between 20-30years are $\{19(76.00 \%), 9(60.00 \%)$ and $4(26.67 \%)\}$ respectively. There was a highly significant difference between three PCOS groups $(\mathrm{P}<0.001)$.In age more than 30 years were $\{5(20.00 \%), 6(40.00 \%)$ and 11 $(73.33 \%)\}$ respectively. Statistical analysis showed a highly significant difference $(\mathrm{P}<0.01)$ between three groups of PCOS (Table -1).

Table 1:-Distribution of Study According to Age /per Group of PCOS

\begin{tabular}{|l|l|l|l|l|}
\hline \multirow{2}{*}{ Age group (year) } & The group & Antagonist & Short Agonist & P-value \\
\cline { 2 - 5 } & Long Agonist & $0(0.00 \%)$ & $0(0.00 \%)$ & $0.469 \mathrm{NS}$ \\
\hline Less than 20 & $1(4.00 \%)$ & $9(60.00 \%)$ & $4(26.67 \%)$ & $0.0001 * *$ \\
\hline $20-30$ & $19(76.00 \%)$ & $6(40.00 \%)$ & $11(73.33 \%)$ & $0.0001 * *$ \\
\hline More than 30 & $5(20.00 \%)$ & 15 & 15 & --- \\
\hline Total & 25 & $0.0001 * *$ & $0.0001 * *$ & --- \\
\hline P-value & $0.0001 * *$ & & \\
\hline$* *(P<0.01)$. & &
\end{tabular}

\section{Distribution of Study According to Type of Infertility /per Group of PCOS}

In the present study, it was found that $21(84.00 \%)$ of PCOS with long protocol had primary infertility, while 4 $(16.00 \%)$ of them were suffering from secondary infertility. In PCOS patients with Antagonist protocol group 14 $(93.33 \%)$ of them were suffering from primary infertility, while $1(6.67 \%)$ of them were suffering from secondary infertility. Eleven (11) (73.33\%) of Third group of PCOS who with short protocol had primary infertility while 4 $(26.67 \%)$ them were suffering from secondary infertility. So, there were highly significant difference $(\mathrm{P}<0.01)$ in type of infertility among different groups of PCOS. In all groups, the primary infertility highly significant than secondary infertility $(\mathrm{P}<0.01)$ (Table -2$)$. 
Table 2:-Distribution of Study According to Type of Infertility /per Group of PCOS

\begin{tabular}{|l|l|l|l|l|}
\hline \multirow{2}{*}{ Type of infertility } & The group & Antagonist & Short Agonist & P-value \\
\cline { 2 - 5 } & Long Agonist & $14(93.33 \%)$ & $11(73.33 \%)$ & $0.0059 * *$ \\
\hline Primary & $21(84.00 \%)$ & $1(6.67 \%)$ & $4(26.67 \%)$ & $0.0059 * *$ \\
\hline Total & $4(16.00 \%)$ & 15 & 15 & -1 \\
\hline P-value & 25 & $0.0001 * *$ & $0.0001 * *$ & \\
\hline$* *(P<0.01)$. & $0.0001 * *$ & & \\
\hline$*$ =highly significant &
\end{tabular}

\section{Distribution of Study According to Duration of Infertility /per Group of PCOS}

In this study, it was found that $10(40.00 \%)$ of PCOS with long protocol had less than five (5) years of infertility, 13 $(52.00 \%)$ of them were suffering from infertility for 5-10 years and only $2(8.00 \%)$ had infertility for more than ten (10) years. In PCOS patients with Antagonist protocol group only 1 (6.67\%) of them was suffering from infertility for less than 5 years, $12(80.00 \%)$ of them were suffering from infertility for 5-10 years, while $2(13.33 \%)$ of them were suffering from more than ten years infertility. Only 1 (6.67\%) of Third group of PCOS who with short protocol had infertility for less than 5 years, $13(86.67 \%)$ of them were suffering from 5-10 years of infertility and $3(20.00 \%)$ of them had more than 10 years infertility. So, there were highly significant difference $(\mathrm{P}<0.01)$ in duration of infertility (less than 5 years and 5-10 years) among different groups of PCOS while there was significant difference $(\mathrm{P}<0.05)$ in duration of infertility more than 10 years among different groups of PCOS. In all groups, 5-10 years duration of infertility is highly significant than less than 5 and more than 10 years infertility $(\mathrm{P}<0.01)($ Table -3$)$.

Table 3:-Distribution of Study According to Duration of Infertility/per Group of PCOS

\begin{tabular}{|l|l|l|l|l|}
\hline \multirow{2}{*}{ Duration (year) } & The group & Antagonist & Short Agonist & P-value \\
\cline { 2 - 5 } & Long Agonist & $1(6.67 \%)$ & $1(6.67 \%)$ & $0.0001 * *$ \\
\hline Less than 5 & $10(40.00 \%)$ & $12(80.00 \%)$ & $13(86.67 \%)$ & $0.0021 * *$ \\
\hline M-10 & $13(52.00 \%)$ & $2(13.33 \%)$ & $3(20.00 \%)$ & $0.0402 *$ \\
\hline Total than 10 & $2(8.00 \%)$ & 15 & 15 & --- \\
\hline P-value & 25 & $0.0001 * *$ & $0.0001 * *$ & --- \\
\hline$*(\mathrm{P}<0.05), * *(\mathrm{P}<0.01)$. & $0.0001 * *$ & & \\
\hline$*=$ significant; $* *=$ highly significant &
\end{tabular}

\section{Distribution of Study According to Cause of Infertility/per Group of PCOS}

In our study, it was found that $12(48.00 \%)$ of PCOS with long protocol had only PCOS as a cause of infertility while $13(52.00 \%)$ of them had PCOS with male cause. All PCOS patients with Antagonist protocol group 15 $(100 \%)$ had PCOS with male cause. In short agonist protocol group12 $(80.00 \%)$ of them had PCOS only while 3 $(20.00 \%)$ had PCOS with male cause of infertility. So, there were highly significant differences $(\mathrm{P}<0.01)$ in cause of infertility among different groups of PCOS (Table-4).

Table 4:-Distribution of Study According to Cause of Infertility/per Group of PCOS

\begin{tabular}{|c|c|c|c|c|}
\hline \multirow[b]{2}{*}{ Cause } & \multicolumn{3}{|l|}{ The group } & \multirow[b]{2}{*}{$\mathrm{P}$-value } \\
\hline & Long Agonist & Antagonist & Short Agonist & \\
\hline PCOS & $12(48.00 \%)$ & $0(0.00 \%)$ & $12(80.00 \%)$ & $0.0001 * *$ \\
\hline PCOS-Male & $13(52.00 \%)$ & $15(100 \%)$ & $3(20.00 \%)$ & $0.0001 * *$ \\
\hline Total & 25 & 15 & 15 & --- \\
\hline P-value & $0.369 \mathrm{NS}$ & $0.0001 * *$ & $0.0035 * *$ & --- \\
\hline
\end{tabular}

PCOS=Poly Cystic Ovary syndrome; NS=non-significant; $* *=$ highly significant. 
Comparison among the Effects of Different IVF/ICSI Protocols (Long agonist, Antagonist, Short agonist) on the Level of Serum Estradiol (E2)at Day of HCG Injection of Patients with PCOS:

In present study, the mean \pm SE for the level of serum E2 in PCOS groups who underwent long agonist, Antagonist, short agonist protocols were $(1957.18 \pm 110.94,2464.74 \pm 115.87$ and $2184.22 \pm 150.48) \mathrm{pg} / \mathrm{ml}$ respectively. The statistical analysis shows significant difference $(\mathrm{P}<0.05)$ in the level of serum E2 among different groups of PCOS (Table 5).

Table 5:-Comparison among the Effects of Different IVF/ICSI Protocols (Long agonist, Antagonist, Short agonist) on the Level of S-Estradiol (E2) at Day of HCG Injection of Patients with PCOS:

\begin{tabular}{|c|c|c|c|}
\hline \multicolumn{2}{|l|}{ Group } & No. & Mean \pm SE of S- Estrogen $(\mathrm{pg} / \mathrm{ml})$ \\
\hline \multirow{3}{*}{ PCOS } & Long & 25 & $1957.18 \pm 110.94 b$ \\
\hline & Antagonist & 15 & $2464.74 \pm 115.87 \mathrm{a}$ \\
\hline & Short & 15 & $2184.22 \pm 150.48 \mathrm{ab}$ \\
\hline \multicolumn{2}{|c|}{ LSD value } & --- & $365.87 *$ \\
\hline \multicolumn{2}{|c|}{ P-value } & --- & 0.020 \\
\hline \multicolumn{4}{|c|}{$*(\mathrm{P}<0.05)$} \\
\hline
\end{tabular}

\section{Comparison among Different Groups of PCOS in Oocyte and MII:}

The Total Number of Oocytes Retrieved

The mean total number of oocytes \pm SE in PCOS groups who underwent long agonist, Antagonist, short agonist protocols were $(11.48 \pm 0.84,11.80 \pm 1.43$ and $9.46 \pm 1.01)$ respectively. The statistical analysis showed no significant difference $(\mathrm{P}>0.05)$ among the different groups of PCOS (Table 6).

\section{Number of Mature Oocytes (Metaphase II)}

In the current study, the mean number of mature oocytes [metaphase II (MII)] \pm SE in PCOS groups who underwent long agonist, Antagonist, short agonist protocols were $(7.04 \pm 0.54,7.53 \pm 0.73$ and $6.00 \pm 0.72)$ respectively. There was no significant difference $(\mathrm{P}>0.05)$ among the three groups of PCOS patients (Table 6).

Table 6:-Comparison among Different Groups of PCOS in Oocyte and MII

\begin{tabular}{|l|l|l|l|l|}
\hline \multirow{2}{*}{ Group } & \multirow{2}{*}{ No. } & Mean \pm SE & No. of Metaphase II \\
\cline { 3 - 5 } & Long & 25 & $11.48 \pm 0.84$ & $7.04 \pm 0.54$ \\
\cline { 2 - 5 } & Antagonist & 15 & $11.80 \pm 1.43$ & $7.53 \pm 0.73$ \\
\cline { 2 - 5 } & Short & 15 & $9.46 \pm 1.01$ & $6.00 \pm 0.72$ \\
\hline LSD value & --- & $3.116 \mathrm{NS}$ & $0.893 \mathrm{NS}$ \\
\hline P-value & --- & 0.302 & \multicolumn{2}{l}{} \\
\hline NS: Non-significant. & \multicolumn{4}{l}{} \\
\hline
\end{tabular}

All values are expressed as mean \pm SE (Standard Error). PCOS=Poly Cystic Ovary syndrome; (MII=Metaphase II) P>0.05 NS: Non-significant.

\section{Distribution of Study According to Day of ET/per Group}

In the current study, embryo transfer was done in day two in PCOS groups who with long agonist, Antagonist, short agonist protocols were $\{8(32.00 \%), 4(26.67 \%)$ and $10(66.67 \%)\}$ respectively. There was a high significant difference $(\mathrm{P}<0.001)$ among the three groups of PCOS patients. On the other hand, day three embryo transfer were done in PCOS groups who with long agonist, Antagonist, short agonist protocols (14 (56.00\%), 11 (73.33\%) and 5 (33.33\%) respectively. The statistical analysis shows a highly significant difference $(\mathrm{p}<0.001)$ in day three embryo transfer among three groups of PCOS. No embryo transfer was done in three cases of PCOS patients with long protocol, two of them no fertilization occurred because of their husband with sever oligoasthenoteratospermia, while third case patient developed high fever so postponed transfer and freezed embryos (Table 7).

Table 7:-Distribution of Study According to Day of ET/per Group

\begin{tabular}{|l|l|l|l|l|}
\hline \multirow{2}{*}{$\begin{array}{l}\text { Day of } \\
\text { Transfer }\end{array}$} & Embryo & The group & P-value \\
\cline { 3 - 5 } & Long Agonist & Antagonist & Short Agonist & \\
\hline
\end{tabular}




\begin{tabular}{|l|l|l|l|l|}
\hline 0 & $3(13.00 \%)$ & $0(0.00 \%)$ & $0(0.00 \%)$ & $0.0388 *$ \\
\hline 2 & $8(32.00 \%)$ & $4(26.67 \%)$ & $10(66.67 \%)$ & $0.0001 * *$ \\
\hline 3 & $14(56.00 \%)$ & $11(73.33 \%)$ & $5(33.33 \%)$ & $0.0001 * *$ \\
\hline Total & 25 & 15 & 15 & --- \\
\hline P-value & $0.0001 * *$ & $0.0001 * *$ & $0.0001 * *$ & --- \\
\hline$*(P<0.05) * *$ \\
\hline
\end{tabular}

$*(\mathrm{P}<0.05), * *(\mathrm{P}<0.001)$.

$*=$ significant; $* *=$ highly significant

Distribution of Study According to Number of Embryo Transfer /per Group of PCOS

In our study, two embryo transfer was done in PCOS groups who with long agonist, Antagonist, short agonist protocols were $\{1(4.00 \%), 2(13.33 \%)$ and $2(13.33 \%)\}$ respectively. There was significant difference $(\mathrm{P}<0.05)$ among the three groups of PCOS patients. On the other hand, three embryo transfer were done in PCOS groups who with long agonist, Antagonist, short agonist protocols $\{9(36.00 \%), 3(20.00 \%)$ and $6(40.00 \%)\}$ respectively. The statistical analysis shows highly significant difference $(\mathrm{p}<0.01)$ among three groups of PCOS. Four embryo transfer was done in $\{9(36.00 \%), 7(46.67 \%)$ and $5(33.33 \%)$ respectively. There was a significant difference $(\mathrm{P}<0.05)$ among different groups of PCOS .However, five embryo transfer was done in $\{3(12.00 \%), 3(20.00 \%)$ and 2 $(13.33 \%)\}$ respectively. The statistical analysis showed significant difference $(\mathrm{P}<0.05)$ among three groups of PCOS. No embryo transfer was done in three cases of PCOS patients with long protocol as we mentioned above (Table-8).

Table 8:-Distribution of Study According to Number of Embryo Transfer /per Group of PCOS

\begin{tabular}{|l|l|l|l|l|}
\hline $\begin{array}{l}\text { Number } \\
\text { transferred } \\
\text { Embryo }\end{array}$ & \multicolumn{2}{|c|}{ The group } & \multirow{2}{*}{ P-value } \\
\cline { 2 - 5 } & Long Agonist & Antagonist & & \\
\hline 0 & $3(12.00 \%)$ & $0(0.00 \%)$ & $0(0.00 \%)$ & $0.0395^{*}$ \\
\hline 2 & $1(4.00 \%)$ & $2(13.33 \%)$ & $2(13.33 \%)$ & $0.0486^{*}$ \\
\hline 3 & $9(36.00 \%)$ & $3(20.00 \%)$ & $6(40.00 \%)$ & $0.0036^{* *}$ \\
\hline 4 & $9(36.00 \%)$ & $7(46.67 \%)$ & $5(33.33 \%)$ & $0.0402 *$ \\
\hline 5 & $3(12.00 \%)$ & $3(20.00 \%)$ & $2(13.33 \%)$ & $0.0498^{*}$ \\
\hline Total & 25 & 15 & 15 & --- \\
\hline P-value & $0.0001 * *$ & $0.0001 * *$ & -- \\
\hline$*(P<0.05), * *(P<0.01)$. & $0.0001 * *$ & \\
\hline
\end{tabular}

\section{Distribution of Study According to Quality of Embryo /per Group of PCOS}

In current study, Grade I(GI) embryo have been transferred in PCOS groups who with long agonist, Antagonist, short agonist protocols were(16 (64.00\%),12 $(80.00 \%), 11(73.33 \%)\}$ respectively which is statistically highly significant $(\mathrm{P}<0.01)$. While Grade II (GII) embryo have been transferred in PCOS groups who with long agonist, Antagonist, short agonist protocols were $(1(4.00 \%), 0(0.00 \%)$ and0 $(0.00 \%)\}$ respectively which is statistically shows no significant $(\mathrm{P}<0.05)$. Grade I, II embryo transfer was done in $(5(2.00 \%), 3(20.00 \%)$ and $4(26.67 \%)\}$ respectively. There was a highly significant difference $(\mathrm{P}<0.01)$ among three groups of PCOS. No embryo transfer was done in three cases of PCOS patients with long protocol as we mentioned above (Table-9).

Table 9:-Distribution of Study According to Quality of Embryo /per Group of PCOS

\begin{tabular}{|c|c|c|c|c|}
\hline \multirow[b]{2}{*}{ Quality of embryo } & \multicolumn{3}{|l|}{ The group } & \multirow[b]{2}{*}{ P-value } \\
\hline & Long Agonist & Antagonist & Short Agonist & \\
\hline GI & $16(64.00 \%)$ & $12(80.00 \%)$ & $11(73.33 \%)$ & $0.0115 * *$ \\
\hline GII & $1(4.00 \%)$ & $0(0.00 \%)$ & $0(0.00 \%)$ & $0.469 \mathrm{NS}$ \\
\hline GI, GII & $5(2.00 \%)$ & $3(20.00 \%)$ & $4(26.67 \%)$ & $0.0086 * *$ \\
\hline Null. & $3(12.00 \%)$ & $0(0.00 \%)$ & $0(0.00 \%)$ & $0.4020 *$ \\
\hline Total & 25 & 15 & 15 & --- \\
\hline P-value & $0.0001 * *$ & $0.0001 * *$ & $0.0001 * *$ & --- \\
\hline
\end{tabular}

*=significant; **=highly significant; GI=Grade I; GII= Grade II 


\section{Comparison among Different Groups of PCO (Long agonist, Antagonist, Short agonist) in Pregnancy} Outcome:

In this study, $4(16.00 \%)$ out of 25 PCOS patient with long protocol, 3 (20.00\%) out of 15 PCOS patient with Antagonist protocol and 6 (40.00\%) out of 15 PCOS patient with short agonist protocol became pregnant. Statistical analysis shows a high significant difference $(\mathrm{P}<0.01)$ among three groups in pregnancy rate. Short agonist protocol shows high significant difference in pregnancy rate in comperes with long agonist and antagonist protocols (Table 10).

Table 10:-Comparison among Different Groups of PCO (Long agonist, Antagonist, Short agonist) in Pregnancy Outcome

\begin{tabular}{|c|c|c|c|c|}
\hline \multirow[b]{2}{*}{ Pregnancy test-PT } & \multicolumn{3}{|l|}{ The group } & \multirow[b]{2}{*}{$\mathrm{P}$-value } \\
\hline & Long Agonist & Antagonist & Short Agonist & \\
\hline Yes & $4(16.00 \%)$ & $3(20.00 \%)$ & $6(40.00 \%)$ & $0.0028 * *$ \\
\hline No & $21(84.00 \%)$ & $12(80.00 \%)$ & $9(60.00 \%)$ & $0.0028 * *$ \\
\hline Total & 25 & 15 & 15 & --- \\
\hline $\mathrm{P}$-value & $0.0028 * *$ & $0.0028 * *$ & $0.0047 * *$ & --- \\
\hline
\end{tabular}

\section{Disscusion:-}

Before 20 years, GnRH agonists have been the "gold standard" protocol in COS. In recent years it has become more complex, and stressful for the couples due to overlong and complex ovarian stimulation protocols, GnRH antagonists, in contrast, are more often used as second-line agents in patients who are poor responders, in the elderly and in the ones with previous IVF failures. Also, the addition of antagonist protocol to the semi natural cycle IVF protocol reduced the premature LH surge without affecting overall outcome. The short protocol seemed to be an efficient and cost-effective protocol for the poor responders and old age. The present study showed no significant difference in the number of oocyte and MII among different groups of PCOS and significant difference in the serum E2 level which high in PCOS patient underwent antagonist (2464.74 \pm 115.87$)$ and short agonist (2184.22 \pm 150.48$)$ than long agonist protocol $(1957.18 \pm 110.94)$. This finding disagreed with Lainas, et al. $(2010)^{(14)}$ that show high number of oocyte, MII and high estradiol level in long agonist protocol than antagonist. Also disagreed with Jianping, et al. (2015) ${ }^{(15)}$ that found, the number of oocytes retrieved, MII oocytes, and high-quality embryos in the long protocol group were all significantly greater than those in the short protocol group. The results in study differed from other studies may explained by: in our study we used long agonist protocol for young age PCOS and start with low dose of FSH (150 IU) to avoid excessive ovarian stimulation and OHSS while we used short agonist protocol and antagonist protocol in PCOS patients more than 30 years old and start with high FSH dose ( $\geq 225 \mathrm{IU})$ to recruit more follicles. As we know that PCOS patients have good ovarian reserve even with age till forty and they are not poor responders and still have sufficient AFC, in addition we should bear in mind the small sample size and limited power, were may resulted into no significant difference in the number of oocyte and MII and significant difference in the serum E2 level among different groups of PCOS. The present study showed that clinical pregnancy rate is higher in PCOS women underwent short agonist protocol (40\%) than in PCOS women underwent long agonist protocol (16\%) and antagonist protocol (20\%). Kaur, et al. (2012) ${ }^{(16)}$ and the meta-analysis of Griesinger et al. (2006) ${ }^{(17)}$ that compared agonist and antagonist protocol in a total of 305 patients with PCOS, and included four studies. In agreement with the results of the present study, pregnancy rates were not significantly different in the agonist and antagonist groups. It should be noted that the difference observed here in ongoing pregnancy rates between the long agonist and antagonist groups (4\%) is below that arbitrarily accepted as clinically significant (5\%), and is not statistically significant. The results in this study are in disagreement with Jianping, et al. (2015) ${ }^{(15)}$ that demonstrate, the clinical pregnancy rate was significantly higher in the long protocol group than those in the short protocol group. The higher pregnancy rate in our study may due to our using of short agonist protocol in PCOS patients more than 35 years old who are with good ovarian reserve and not poor responder, together with high starting FSH dose ( $\geq 225 \mathrm{IU})$ to recruit more follicles .As mentioned, adequate number of oocyte and MII and high level of E2 were obtained in patient underwent short agonist protocol which result in obtained good quality embryo and high pregnancy rate. Also should bear in mind the small sample size that resulted. 


\section{Referrences:-}

1. Byneil S and Charissa M. Polycystic Ovary Syndrome. Obstet. Gynecol. 2009;114: 936-49.

2. P. Peitsidis, R. Agrawal. Role of vascular endothelial growth factor in women with PCO and PCOS: a systematic review. Reprod Biomed Online, 20 (2010), pp. 444-452.

3. Pelinck MJ, Hoek A, Simons AH and Heineman MJ. Efficacy of natural cycle IVF: a review of the literature. Hum Reprod Update. 2002; 8(2): pp.129- 139.

4. Depalo R, Jayakrishan K, Garruti G, Totaro I; Panzarino M; Giorgino F and Selvaggi LE. GnRH agonist versus GnRH antagonist in in vitro fertilization and embryo transfer (IVF/ET). Reprod Biol Endocrinol. 2012; 10: p.26.

5. Schats R and Huirne JAF. The use of GnRH agonists. In : Gradner DK, Weissman A, Howles CM, Shoham Z, editors. Textbook of Assisted Reproductive Technologies, Volume Two: Clinical Perspectives, 4th ed.London: Informa Health care. 2012. pp.115-23.

6. $\mathrm{Pu} \mathrm{D}, \mathrm{Wu}$ J, and Liu J. Comparisons of GnRH antagonist versus GnRH agonist protocol in poor ovarian responders undergoing IVF. Hum Reprod. 2011; 26: pp.2742-2749.

7. Rotterdam ESHRE/ASRM-sponsored PCOS consensus workshop group :Revised 2003 consensus on diagnostic criteria and long-term health risks related to Polycystic ovary syndrome (PCOS), Hum Reprod 19 .2004; pp. 41-47, Fertile Steril.; 81: 19-25. 11.

8. Hugues JN, Cédrin-Durnerin I. Endocrine characteristics of ART Cycles. In : Gradner DK, Weissman A, Howles CM, Shoham Z ,editors. Textbook of Assisted Reproductive Technologies, Volume Two: Clinical Perspective.4th ed. London: Informa Health care; 2012. pp.99-114.

9. Elder K, Dale B. In-Vitro Fertilization.3rded. New York: Cambridge University Press. 2011. pp.19-27.

10. Zegers-Hochschild F, Adamson GD, de Mouzon J, et al. The International Committee for Monitoring Assisted Reproductive Technology (ICMART) and the World Health Organization (WHO) Revised Glossary on ART Terminology.2009. Hum. Reprod. 2009; 24: 2683-7. 87.

11. Al-Inany H, and Aboulghar M. GnRH antagonist in assisted reproduction:a Cochrane review. Hum Reprod. $2002 ; 17: 874-885$.

12. Schats R, Huirne JAF. The use of GnRH agonists. In : Gradner DK, Weissman A, Howles CM, Shoham Z, editors. Textbook of Assisted Reproductive Technologies, Volume Two: Clinical Perspectives, 4th ed.London: Informa Health care. 2012. pp.115-23.

13. SAS. 2012. Statistical Analysis System, User's Guide. Statistical. Version 9.1th ed. SAS. Inst. Inc. Cary. N.C. USA.

14. Lainas TG, Ioannis S, Zorzovillis IZ, Petsas GK, Lainas GT and Alexopoulou E. Flexible GnRH antagonist protocol versus GnRH agonist long protocol in patients with polycystic ovary syndrome treated for IVF: A prospective randomized controlled trial (RCT) Hum Reprod. 2010;25: pp.683-9

15. Jianping Ou, Weijie Xing, Yubin Li, Yanwen Xu and Canquan Zhou. Short versus Long GonadotropinReleasing Hormone Analogue suppression Protocols in IVF/ICSI Cycles in Patients of Various Age Ranges. J. plos. Org. July 24, 2015.

16. Kaur H, Krishna D, Shetty N, Krishnan S, Srinivas M and Rao KA. A prospective study of GnRH long agonist versus flexible GnRHantagonist protocol in PCOS: Indian experience. J Hum Reprod Sci. 2012; 5: pp.181-6.

17. Griesinger G, Diedrich K, Tarlatzis BC and Kolibianakis EM. GnRH-antagonists in ovarian stimulation for IVF in patients with poor response to gonadotrophins, polycystic ovary syndrome, and risk of ovarian hyperstimulation: A meta-analysis. Reprod Biomed Online. 2006; 13: pp.628-38. 